\title{
HIGHLIGHTS
}

BLADDER CANCER

\section{Does augmentation cystoplasty increase the risk of bladder cancer?}

The increased risk of bladder cancer associated with augmentation cystoplasty in patients with bladder dysfunction has been disputed by new research. "This study points out that the bladders we augment are not 'normal' and are at an underlying risk of malignancy even in the absence of a bladder augmentation," explains senior author Douglas Husmann from the Mayo Clinic, Rochester, USA.

Augmentation cystoplasty is used to manage bladder dysfunction owing to congenital or neurological abnormalities. Previous findings have shown that bladder augmentation increases the risk of malignancy, and the benefits and disadvantages of this procedure are currently debated.

Husmann and colleagues examined a 1986-2010 prospective registry of patients with bladder dysfunction. To make direct comparisons between groups, the investigators matched patients who had undergone enterocystoplasty with control patients who were treated with intermittent catheterization (with or without anticholinergic therapy) according to type of bladder dysfunction, age and sex. 153 patients had undergone enterocystoplasty (of whom 109 had ileal cystoplasties and 44 had colonic cystoplasties). Nearly half of these patients had initial treatment elsewhere and had been referred to the study center for long-term management.

No difference in the incidence of bladder cancer was observed between the control and enterocystoplasty groups ( $2.6 \%$ versus $4.6 \%)$. Moreover, in those diagnosed with bladder cancer, age at diagnosis, cancer stage, mortality rate and survival did not differ between the two treatment populations. Additionally, patients on chronic immunosuppression, irrespective of treatment with augmentation cystoplasty, had a higher incidence of bladder cancer than those who were not immunosuppressed.

Husmann notes that these findings could affect recommendations for surveillance of malignancy in patients who have undergone augmentation

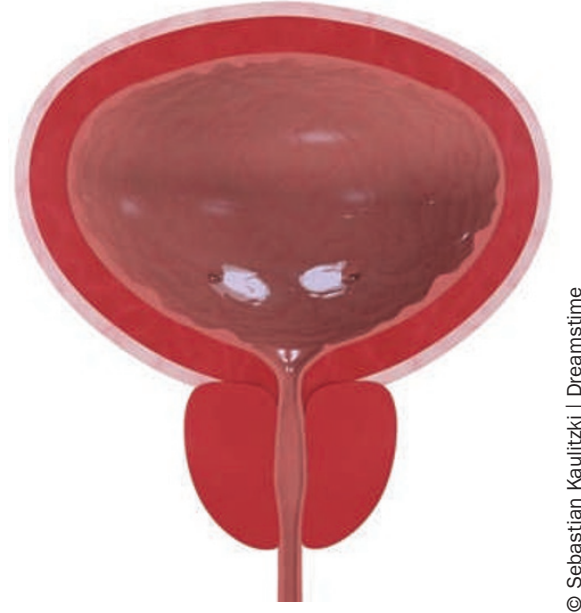

cystoplasty and that inherent risks of cancer must be remembered, especially when using autologous bladder cells for tissue engineering.

Katrina Ray

Original article Higuchi, T. T. et al. Augmentation cystoplasty and risk of neoplasia: fact, fiction and controversy. J. Urol. 184, 2492-2497 (2010) 